\title{
Advantages and disadvantages morphological methods in engineering
}

\author{
Dmitry Rakov* \\ Mechanical Engineering Research Institute of the Russian Academy of Sciences (IMASH) Moscow, \\ 101990, Russia
}

\begin{abstract}
The morphological methods are successful analytical and practical tools for development and choice of new engineering solutions. They can be successfully used in the conceptual and preliminary design stages of the development of new systems, products and technologies. The article discusses the scope of application, classification, advantages and disadvantages of this approach. The design process consists of two stages structural synthesis and parametric optimization. The morphological approach is used for the structural synthesis stage. Structural synthesis generates a significant number of variants. This stage is the most important tool for achieving the high characteristics of the systems being designed. The article discusses the ratios between parametric, structural and structural-parametric methods of synthesis. The possibility of computerizing the stage of structural synthesis on morphological approach is discussed.
\end{abstract}

\section{Introduction}

The new engineering systems (ES) search should be conducted from the point of view of system analysis, the main tasks of which are - decomposition, analysis and system synthesis. The analysis is carried out to establish the properties of ES and the patterns of its functioning. The task of synthesis is to develop the ES model, determine its structure (structural synthesis) and parameters (parametric synthesis or parametric optimization) that ensure the efficient functioning of the system and achieve the goals set.

Problems like the finding of optimal ES belong to systems theory. Systems theory is the interdisciplinary study of systems. Every system is delineated by its spatial and temporal boundaries, surrounded and influenced by its environment, described by its structure and purpose or nature and expressed in its functioning. In terms of its effects, a system can be more than the sum of its parts if it expresses synergy or emergent behavior [1]. The main tasks solved by the theory of systems are:

During the analysis stage:

- $\quad$ Separating the system from the environment and determining its area of existence;

- $\quad$ Determining the functions of the system;

\footnotetext{
* Corresponding author: rdl@mail.ru
} 
- Determining the overall structure of the system, its elements, the relations and the relationship between them;

- $\quad$ Determining the patterns of the system's existence and development;

- Issues of environmental influence;

- $\quad$ Issues of the system's impact on the external environment;

- $\quad$ Modeling and building a system model.

During the synthesis stage:

- $\quad$ Generating a set of variants;

- Determining the rational structure of the system and choosing the best;

- Determining the interaction between subsystems and elements;

- Choosing and analyzing algorithms and scenarios for the system.

\section{Morphological approaches}

The most common approaches among the algorithmic methods is morphological analysis $[2,3]$. By frequency of use, morphological methods are the first among ranks of discursive approaches. Morphological synthesis is regarded as a methodology to problem solving. The morphological analysis is a method (developed by F. Zwicky) to explore all possible solutions of a multi-dimensional, non-quantified problem complex [4].

The morphological box method is performed in the following order $[5,6]$.

- Give a precise problem formulation and a general description of the research object;

- $\quad$ Form important attributes (properties, functions) of the object. The set of features ensures the existence and functioning of the object of research;

- $\quad$ Each attribute is assigned possible implementation options;

- Set of generated variants is reduced to a morphological matrix or morphological box;

- Choice and analysis solutions from the morphological box and determine their value.

\section{Ratios between parametric, structural and structural- parametric synthesis}

The complex system design is usually viewed from two perspectives - macro-design and micro-design.In macrodesign (external design) the functional and structural issues of the system as a whole are solved. The macro-design includes main sections (Fig.1):

- $\quad$ Setting the goals of the system and the range of tasks it solves;

- Description of the factors on the system that must be taken into account in the development of the system;

- Choosing an indicator or a group of system performance indicators.

The design of a system (device, process) is a set of two main tasks: the definition of (a) the structure (structural synthesis) and (b) of parameter range for the synthesized structure (parametric synthesis or parametric optimization) [4]. The solution strategies for these two tasks are different. The parametric synthesis task is usually reduced to determine solutions satisfying the metric criteria, making them formally resolved. In contrast, the task of structural synthesis is absolutely different and cannot be generally allocated to the class of formally solvable problems. The structural synthesis result is the choice of the rational structure of the object (i.e. ES). This requires to work with uncertain structural connections, non-metrical attributes of the structure elements and quality criteria. The objective function of a structural synthesis does not correspond to the main requirements of usual optimization 
methods because (1) it is discontinuous or cannot always be determined; (2) it exists in operator notation; (3) it is not based on analytical expressions; (4) it is not differentiable, not unimodal, not separable, and not additive [1]. The solution of the structural synthesis task is the main and exclusive subject of the researcher's creative activity [4].

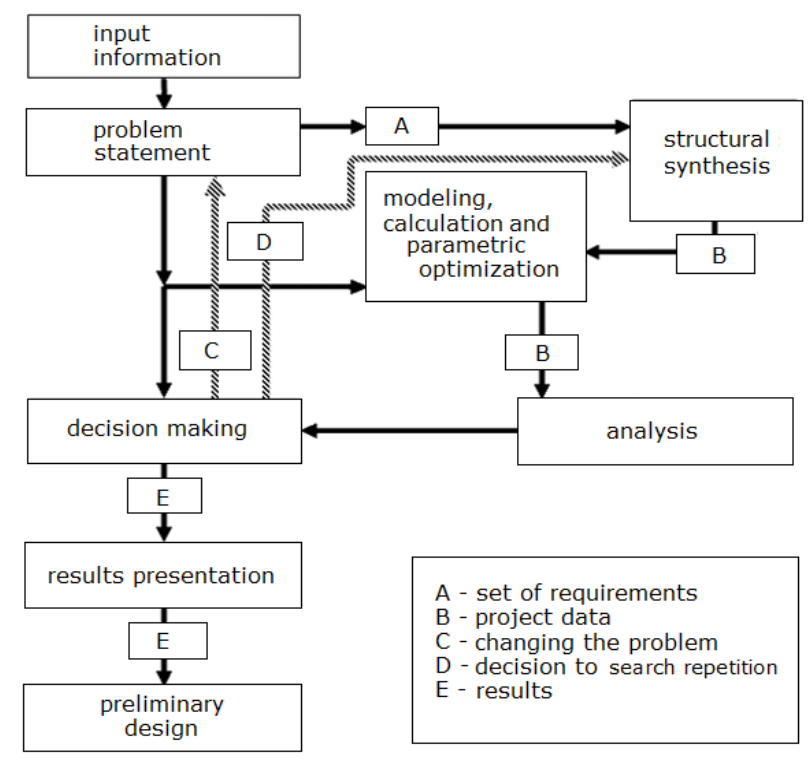

Fig. 1. Macro description of the design process.

Simple systems consist of a small number of elements with a small range of possible states. These systems consist of a small set of simple connections connecting these elements. In part, there is no modeling and optimization of such systems.

The object of design is products in the form of a device, technology, etc. New innovative complex systems generally have no close analogues, i.e. they are characterized by incomplete information and data. As a result, the design process is iterative.

Structural synthesis generates a significant number of variants. This stage is the most important tool for achieving the high characteristics of the systems being designed. The structural synthesis stage is a difficult formalized design phase. It is based on heuristic procedures and peer review methods and is the main activity of the developer. The result of the stage is the choice of the best variants. But incompleteness and lack of unambiguous data and imperfection of structural synthesis methods does not allow to determine unequivocally the best variant. The choice of the best structure boils down to the search for a rational structure, and the conclusions are advisory. Selected structure (structures) are the basis for subsequent modeling and parametric synthesis or parametric optimization. the basis of the diagram or sketch of the device being developed, which allows you to better present it, facilitates the selection and construction of the calculation scheme (calculation model).

Once the parameters of the system have been determined, it becomes possible to test earlier hypotheses and confirm the correctness of the structure choice. The accuracy of the models and solutions that are chosen ensures that the results are accurate.

Depending on the complexity of the tasks, they use (Fig.2, Table 1):

- $\quad$ parametric synthesis (optimization);

- $\quad$ structural synthesis; 
- $\quad$ structural and parametric synthesis.

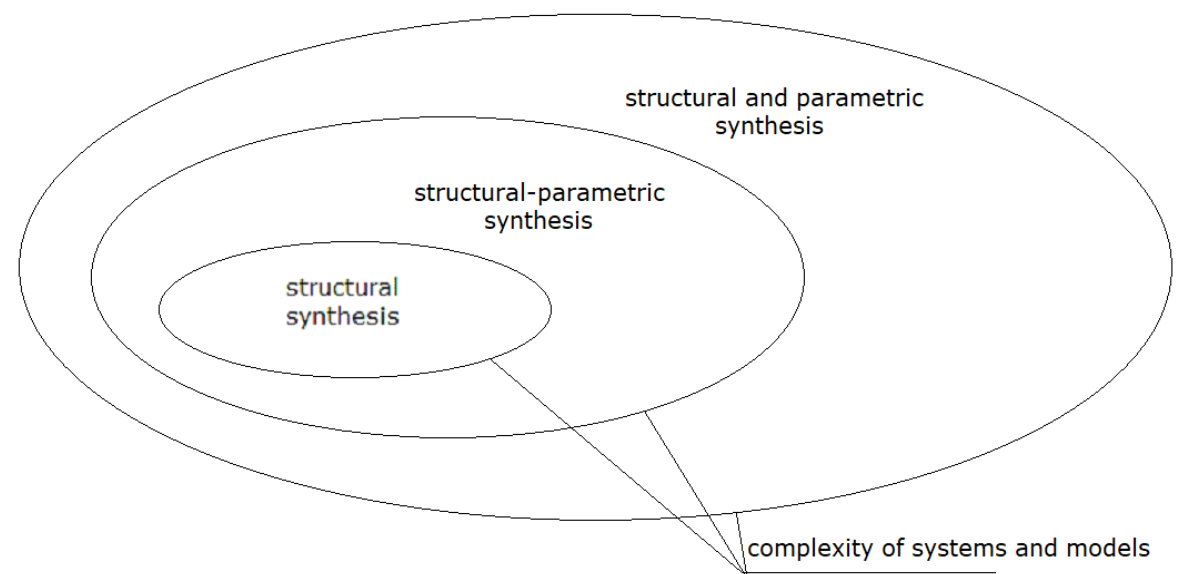

Fig. 2. The dependencies models from the tasks complexity.

Table 1. Ratios between parametric and structural synthesis and structural-parametric synthesis.

\begin{tabular}{|l|l|l|l|l|}
\hline Stage & $\begin{array}{l}\text { Parametric } \\
\text { Optimization }\end{array}$ & $\begin{array}{l}\text { Structural } \\
\text { synthesis }\end{array}$ & $\begin{array}{l}\text { Structural- } \\
\text { parametric } \\
\text { synthesis }\end{array}$ & $\begin{array}{l}\text { Structural } \\
\text { synthesis and } \\
\text { parametric } \\
\text { synthesis }\end{array}$ \\
\hline Structural synthesis & - & + & + & + \\
\cline { 1 - 3 } $\begin{array}{l}\text { Parametric synthesis } \\
\text { (modeling) }\end{array}$ & + & - & + & + \\
\hline Systems & $\begin{array}{l}\text { simple } \\
\text { systems, } \\
\text { complex } \\
\text { systems }\end{array}$ & $\begin{array}{l}\text { simple } \\
\text { systems }\end{array}$ & $\begin{array}{l}\text { complex } \\
\text { systems }\end{array}$ & $\begin{array}{l}\text { complex } \\
\text { systems }\end{array}$ \\
\hline
\end{tabular}

In parametric optimization, the structure of the system is considered fixed. In the process of optimization, the parameters of the elements that make up the structure of the projected system change.

In structural-parametric synthesis (optimization) - both parameters and structure of the system change (Fig.3) [7,8]. The target function for each structure of the system will be unique, and an algorithm of its automatic formation is needed. The structural-parametric optimization can be used in a limited number of cases and for relatively simple research systems with a low degree of innovation. The condition for using this type of optimization is the presence of mathematical models for both structure and parameters. As a rule, when researching new engineering systems, it is impossible to build adequate models for structural synthesis and parametric optimization at the same time.

Structural synthesis is used for simple systems that do not require subsequent modeling and parametric optimization (Fig.4). It is also used in the study of complex systems (Fig.1). In the first stage, a number of rational variants of structures are synthesized and fixed. The second stage is based on parametric models and optimization. 


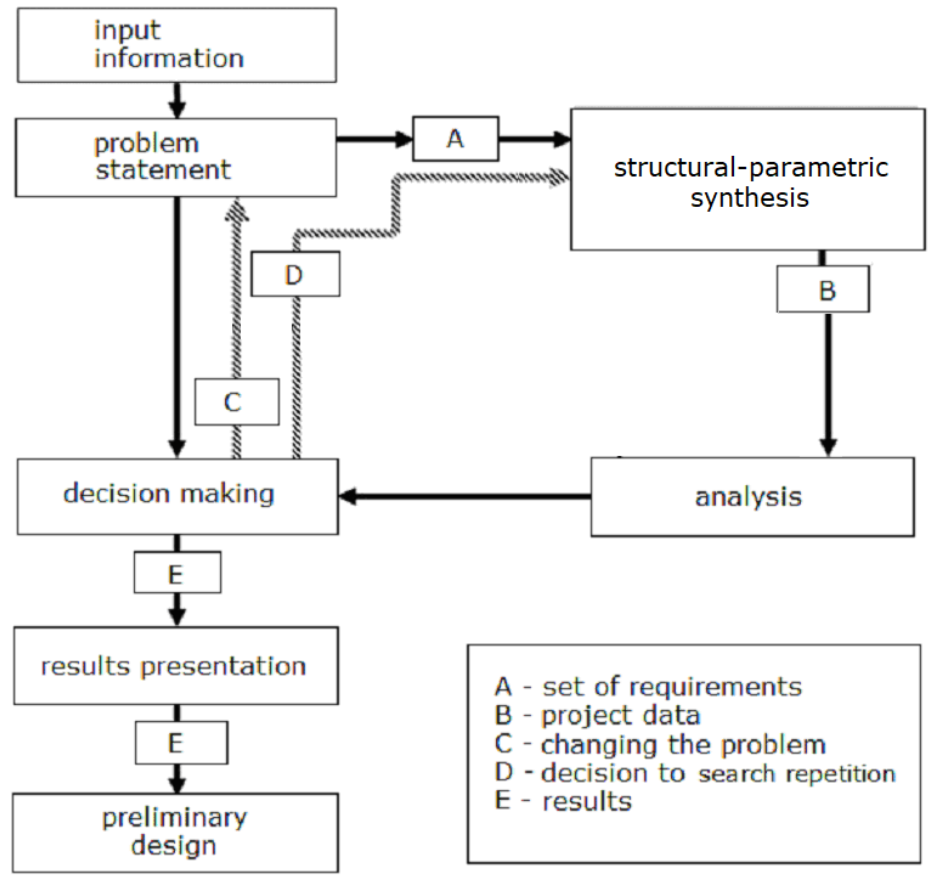

Fig. 3. Macro description of the design process with structural-parametric synthesis (optimization).

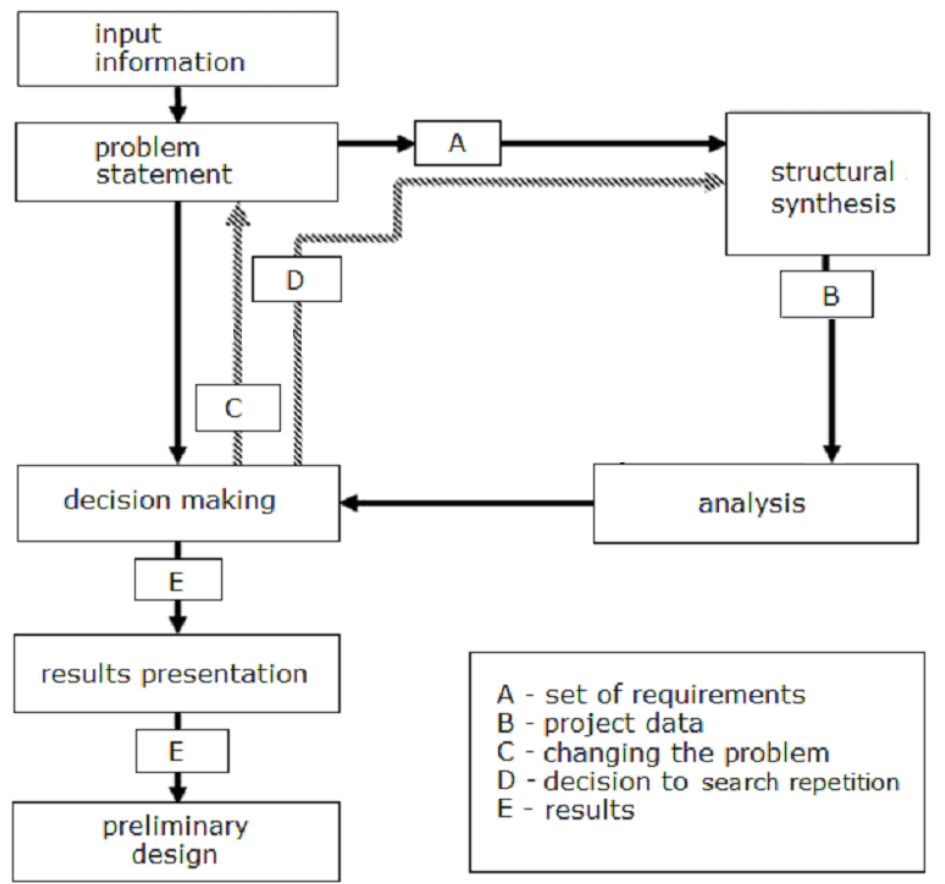

Fig. 4. Macro description of the design process with structural synthesis. 
The complex system has the following main features:

- A large number of mutually connected and interacting elements;

- Emergent behavior;

- $\quad$ Complexity of functions performed by the system, versatility;

- Multidimensionality of the system;

- Variety of possible forms (diversity of structure);

- Multicriteriality;

- Diversity of elements nature;

- Multiple changes in the structure and composition of the system

- Hierarchical;

- Control;

- $\quad$ Interacting with the external environment;

- $\quad$ impact on the external environment;

- $\quad$ to operate in the face of random factors and uncertainty;

- $\quad$ Flexibility and adaptability.

Structural synthesis tasks belong to the class of hard-formed tasks. Also the traditional design methods do not guarantee the optimal of the system being developed. But with the help of program, it is possible to overdo a large number of structures and the possibility of finding the most rational structure seems highly likely [9].

\section{Advantages and disadvantages of morphological methods}

The undoubted advantages of the morphological method include its ability to give valuable results in relation to the original purpose of the search.

The advantages of morphological approaches:

- Methods can be a convenient means of streamlining information related to the research area of knowledge, a means of multi-pro vial of information arrays in information and search engines;

- The morphological matrix can serve as an effective tool for predicting the development of ES. Forecasting is understood here as a search for promising ES classes, not individual solutions;

- There is a fundamental possibility of combining morphological tables with various transformational techniques and procedures of brainstorming and its varieties, synectics, method of focal objects, generalized heuristic method, functional-value analysis, method of functional design, etc.;

- The morphological approach identifies many potential engineering solutions. This stimulates the imagination and leads to creative methodical work. Computerization greatly enhances this incentive;

- The relative ease (quickly) of obtaining a large number of different solutions by constructing a morphological matrix and conducting combinator's analysis;

- The organization and focus of the search for new solutions due to the subordination of the original goal (the final result requirements) and the overall structure of the object;

- The reliability of the process, the wide scope of the task due to the system research of objects.

The disadvantages of classical morphological approaches:

- The time-consuming choice of many options is the best solution in terms of the requirements; 
- The lack of an effective, practical, universal way to objectively assess the effectiveness of a problem option;

- Lack of direct recommendations on the use of specific techniques to revitalize creative thinking at the stages of solving the problem (problem);

- In some cases, the inability to bust and analyze all possible options - the power of the morphological set can be enormous.

Some of the major problems of application of classical methods of morphological analysis are: poor access to support software which can address the combination explosion generated by multi-parameter problem spaces inherent in the use of morphological analysis; insufficiently flexible processes that address users' operational constraints; seen to be overly generic, disguising identification of specific application areas of interest [10].

\section{Conclusion}

The undoubted advantages of the morphological method include its ability to give valuable results in relation to the original purpose of the search. It allows to search for new engineering solutions during the conceptual design phase. In the article considered the ratios between parametric, structural and structural-parametric methods of synthesis. The possibility of computerizing the stage of structural synthesis on morphological approaches is discussed.

\section{References}

1. V.Mishin, M. Osin, Introduction to Aircrafts Design (Moscow, Mashinostroenie, 1978)

2. M. Levin, Modular System Design and Evaluation (Berlin,Springer International Publishing 2015)

3. T.Ritchey, General morphological analysis as a basic scientific modelling method, Technological Forecasting and Social Change, 126, pp. 81-91, (2018)

4. A. Bardenhagen, D. Rakov, Analysis and Synthesis of Aircraft Configurations during Conceptual Design using an Advanced Morphological Approach (Darmstadt, Deutsche Gesellschaft für Luft- und Raumfahrt - Lilienthal-Oberth e.V., 2019)

5. F. Zwicky, Discovery, Invention, Research - Through the Morphological Approach, (Toronto, The Macmillan Company, 1969)

6. F. Zwicky, The Morphological Method of Analysis and Construction, Courant Anniversary Volume, pp. 461-70, (1948)

7. I. Statnikov, G. Firsov, Processing of the results of the planned mathematical experiment in solving problems of research of dynamics machines and mechanisms. International Multi-Conference on Industrial Engineering and Modern Technologies (FarEastCon), pp. 1-6, (2018)

8. R. Statnikov, J. Matusov, A. Statnikov, Multicriteria Engineering Optimization Problems: Statement, Solution and Applications, J Optim Theory Appl, 155, pp. 355375 (2012)

9. D. Rakov, Okkam - Advanced Morphological Approach as Method for Computer Aided Innovation (CAI), MATEC Web of Conferences 298, ICMTMTE 2019, 00120 (2019)

10. B. Garvey, Combining quantitative and qualitative aspects of problem structuring in computational morphological analysis, (London, Imperial College, 2016) 